D out of your grant in three months."

And costs aside, the cloud will probably never suit some computer projects, such as 'deep learning' networks that seek to mimic how the human brain learns. Adam Coates, a computer scientist at Stanford University in California who is involved in such work, says that these systems can require rapid information transfer between billions of connections - something not possible with the cloud. Instead, Coates relies on a dedicated on-site computing cluster. "Having that very high-speed communication is key," he says. "We want vast amounts of computation, but we don't really care about elasticity."

The cloud's dependability is also a concern, says Ken Birman, a computer scientist at Cornell. "It isn't known for being secure, and it isn't known for being extremely reliable.” But not all researchers require foolproof data encryption or super-fast, reproducible computations.

For example, CERN, Europe's particlephysics laboratory near Geneva in Switzerland, assembled an in-house cloud to handle the data generated by the Large Hadron Collider. "The CERN data are public data, so we don't have any security concerns," says Tim Bell, who

\section{ROOM TO GROW}

A 2013 survey of participants in 80 research projects powered by cloud computing reveals the flexibility it affords for handling data ebb and flow.

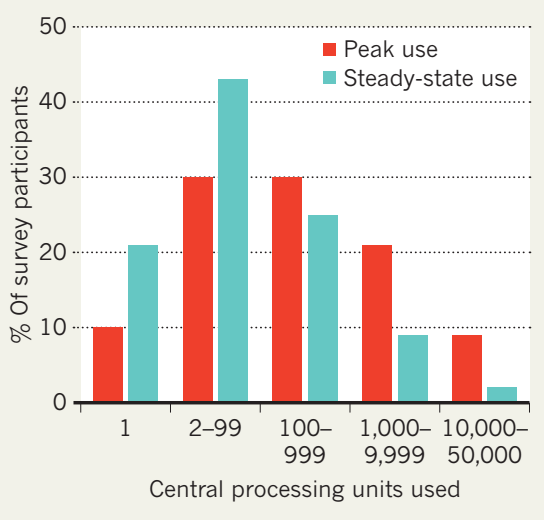

directs the centre's infrastructure and operating services. Instead, CERN focused on providing physicists with an efficient computing platform. "In the past, when they asked for physical hardware, they were waiting for weeks," Bell says. "Now they can ask for a virtual machine and get something in the time it takes to have a cup of coffee."

Universities are also getting into the cloud business. At Cornell, a subscription to Red Cloud costs $\$ 400$ for 8,585 processing hours; for off-campus scientists, the same subscription is $\$ 640$. Such on-campus services often appeal to researchers who are not ready for the do-ityourself nature of commercial providers, which often requires expertise in programming, testing and debugging code. By contrast, Cornell cloud specialists are on site to help researchers using Red Cloud. "The thing you can't get with commercial clouds is hand-holding," Lifka says.

Meanwhile, companies such as Microsoft have set up cloud training specifically for academics, addressing issues such as data sharing and security, scientific reproducibility and how funding agencies may view the cloud. "A lot of the training and education content was tuned to a business audience. That meant the on-ramp for researchers was a bit more tricky," says Daron Green, senior director of Microsoft Research Connections. "We realized there was pretty much a latent demand within the research community." -

\title{
Biomedical institute opens its doors to physicists
}

\section{The development is part of a growing trend to tap physics expertise.}

\section{BY ELIZABETH GIBNEY}

$\mathrm{F}$ rom using soap bubbles when modelling cell division, to applying synchronized clocks to understand embryonic development, physics is becoming an increasingly effective tool for biologists. Now the field is to be a focus of a major new biomedical research hub in London, the Francis Crick Institute.

The $£ 650$-million (US\$1.1-billion) centre - named after the co-discoverer of the structure of DNA, and a physicist-turned-biologist himself — will harness theoretical and experimental approaches from the physical sciences for medical research. When the institute opens in 2015, as much as one-fifth of its 1,250 staff will be physicists, chemists, mathematicians and engineers. They will be tasked with helping biomedical staff to understand why diseases develop and to find new ways to treat them.

The institute is a collaboration between the UK Medical Research Council (MRC), two charities - the Wellcome Trust and Cancer Research UK - and three London universities:
Imperial College London, King's College London and University College London (UCL). The partners are already exploring the potential of bringing diverse fields together, with a workshop next week on astronomy and biomedical imaging. "When you see stars in the sky and you have to analyse their pattern and distribution and understand how they work, it's the same as looking down a microscope - the algorithms you use might well be very similar," says Jim Smith, a Crick board member and a director of the MRC's National

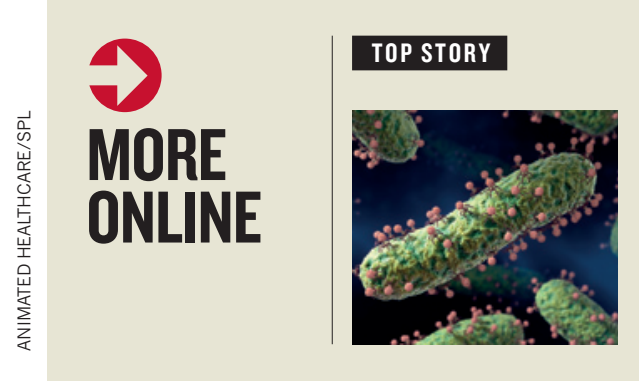

Microbes use nonstandard gene coding in nature go.nature. com/9a3zbc

\section{MORE NEWS}

- Healthy placenta plays host to bacterial flora go.nature.com/Imht8k - How atoms form giant threesomes go.nature.com/yjeaxh

- NASA might end Spitzer telescope in bid to save other missions go.nature. com/geulakr

\section{NATURE PODCAST}

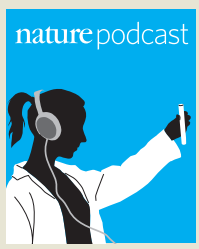

Protons magnetism; antibiotic resistance; and fume-free stoves nature.com/nature/ podcast 


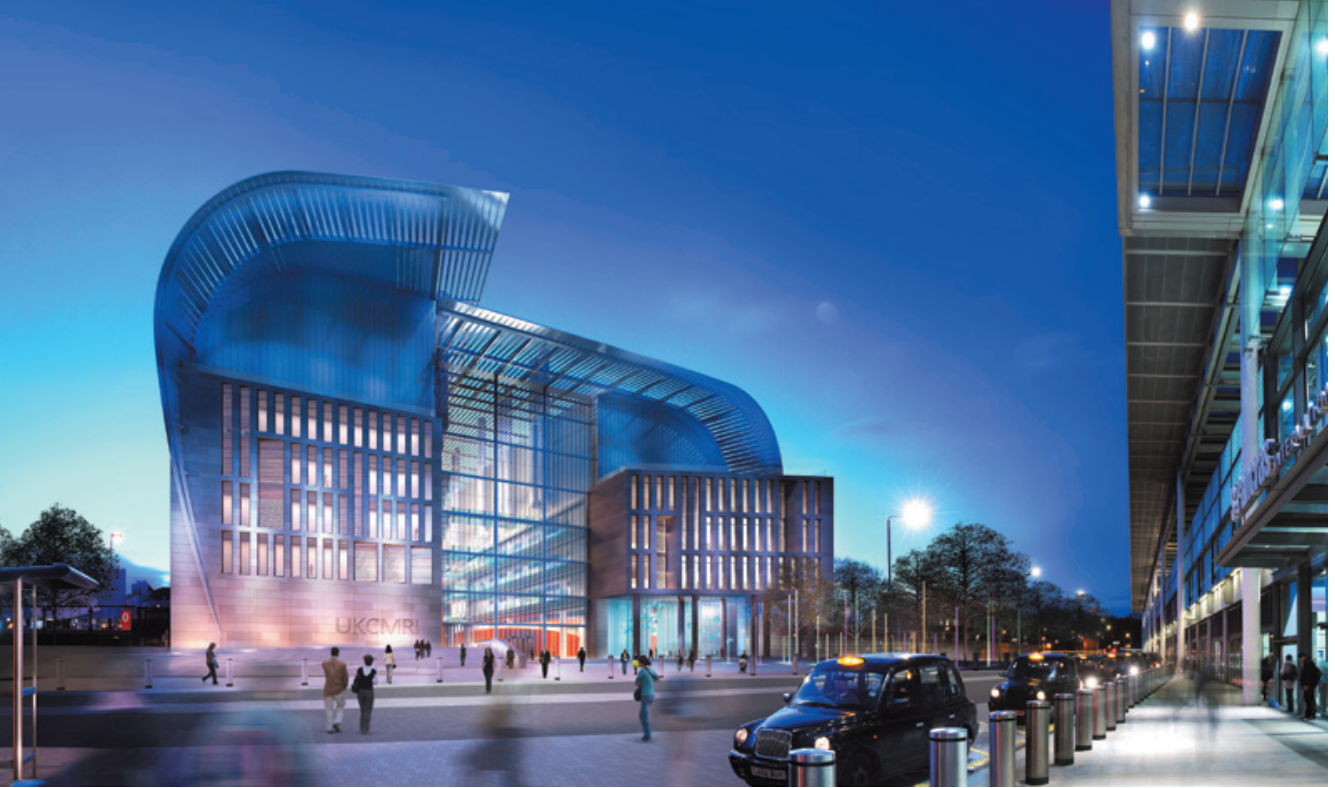

The interdisciplinary Francis Crick Institute will open in central London in 2015.

Institute for Medical Research, which will be subsumed into the Crick.

The term biophysics has in the past often been associated with the study of protein structure or ion-channel function. But the Crick is part of a movement that is taking physics in biology in a new direction. This could, for instance, involve modelling the emergence of shapes and patterns in biological systems across different scales, says Ewa Paluch, a cell biophysicist at UCL. "How do you go from molecules to cells, cells to tissues and organisms to groups of organisms? All these involve crossing scales, and are problems that can't really be understood without a model, and that's where physics comes in."

The approach could reveal how microscopic processes give rise to cell- and tissuescale behaviours, and has been driven partly by improved imaging techniques and growth in the physics of 'soft matter', says Paluch, who will head a new Institute for the Physics of Living Systems at UCL, opening this autumn.

The application of physics in biology has been on the rise in the past decade, with increases in cross-discipline conferences, graduate courses and funding. Germany's Max Planck Society was among the first to support the approach when two of its centres, the Max Planck Institute of Molecular Cell Biology and Genetics and the Max Planck Institute for the Physics of Complex Systems, both in Dresden, began collaborating in 2002. The United States has also been a pioneer, says Alexander van Oudenaarden, a quantitative biologist at the Hubrecht Institute in Utrecht, the Netherlands. Until 2012, he was director of one of 12 Physical Sciences-Oncology Centers founded in 2009 by the National Cancer Institute, part of the US National Institutes of Health (NIH). Such was their success, for example in breastcancer research, that last month the NIH opened a call for a new round of centres.

It might be argued that the emergence of the physics of biology is more of a renaissance than a new development, notes Paluch. Before the 'molecular revolution' of the 1950s, which

refocused much biological research on genetics and molecular biology, physics played a bigger part in the field, she says - as demonstrated by Crick's involvement in the discovery of the DNA helix. But the shift sidelined physics.

Now the field can help biologists to handle the huge amounts of data they generate, image biological organisms in new ways and design materials, says Richard Treisman, director of Cancer Research UK's London Research

\section{“It's not always easy to get a biophysics paper published. It's a problem we face constantly."}

Institute. "There are a huge number of issues where, like it or not, you're dragged kicking and screaming into the physical sciences," he says.

Such sentiments

hint at a potential drawback in the coming together of disciplines - a clash of cultures. Biologists and physicists can sometimes speak different languages, so the communities at the Crick will need to learn to talk to each other.

The institute aims to smooth that process, says Smith. Whole groups from the physicalscience fields will be able to take sabbaticals or secondments within the centre, and the facility has even been designed architecturally to encourage chance encounters that could stimulate ideas.

Biomedical funders have also recognized the benefits of engaging with physics. As part of its new research strategy, Cancer Research UK last month announced a $£ 5$-million fund dedicated to collaboration with the physical sciences, a decision it took after reviewing the effectiveness of such approaches.

This kind of initiative is welcome, says Paluch. Despite the rise in joint grants, convincing peer-review panels to fund interdisciplinary work is not straightforward, she adds. Nor is getting such work published, because many biology journals expect different kinds of mechanistic explanations from those usually put forward in physics. "It's not always easy to get a biophysics paper published. It's a problem we face constantly," she says. 